\title{
Die anomale Dispersion an den Linien der gelbgrünen Bromdampfbanden*
}

\author{
Von Helmut Faissner
}

\author{
Aus dem I. Physikalischen Institut der Universität und dem Institut für Physik im \\ Max-Planck-Institut für med. Forschung Heidelberg \\ (Z. Naturforschg. 8a, 633-645 [1953]; eingegangen am 31. Juli 1953)
}

\begin{abstract}
Die an den Einzellinien der gelbgrünen Bromdampfbanden auftretende anomale Dispersion wurde quantitativ untersucht. Als Grundlage der Messungen diente die Hakenmethode nach Roschdestwensky. Die komparatorische Ausmessung der Hakenabstände wurde in einer Reihe der zwischen 5600 und $5800 \AA$ gelegenen $\mathrm{Br}_{2}{ }^{79},{ }^{81}$-Banden des $\left(v^{\prime \prime}=1\right)$ - und $\left(v^{\prime \prime}=0\right)$-Bandenzugs und zum Teil bei verschiedenen Bromdrucken vorgenommen. Die Abhängigkeit der gemessenen Dispersionselektronendichten $\mathfrak{N}_{j k}$ vom Druck ist im Rahmen der Meßgenauigkeit linear. Weiter wurde eine indirekte Methode zur Bestimmung der Hakenabstände entwickelt. Sie beruht auf der Zusammensetzung der an den Einzellinien hervorgerufenen Interferenzstreifen-Verzerrungen zu charakteristischen, ,Bögen“ genannten Schattenphänomenen und deren Verschwinden mit wachsendem Druck. Das Verschwinden der Bögen wurde mit Hilfe der Lorentzschen Stoßdämpfungstheorie geklärt und quantitativ beschrieben. Die Messungen wurden mit Steigerung des Eigendrucks und Zusatz von Wasserstoff an den zwischen 5200 und $5800 \AA$ gelegenen Banden der vorhin genannten Bandenzüge durchgeführt. Die nach den verschiedenen Methoden erhaltenen Oszillatorenstärken stimmen gut überein. Die Abhängigkeit der Linienintensitäten von den Schwingungsquantenzahlen im oberen und unteren Elektronenzustand zeigt den nach dem Franck-Cond on-Prinzip zu erwartenden Verlauf.
\end{abstract}

\section{Problemstellung}

$\mathrm{D}$ e anomale Dispersion ist an den Linien atomarer Spektren bereits eingehend untersucht worden. Am besten hat sich dabei die interferometrische Methode bewährt, die von $\mathrm{Puccianti}^{2}$ eingeführt und von Roschdestwensky ${ }^{3}$ zur Hakenmethode verfeinert wurde. Roschdestwensky gelang es so, an den Natrium-D-Linien die klassische Dispersionsformel mit einer Genauigkeit von $2,5 \%$ zu bestätigen. Die Untersuchungen wurden von ihm und seiner Schule ${ }^{4-6}$ später auf weitere Serienglieder und andere Alkalidämpfe ausgedehnt. Auch über andere Metalldämpfe liegen mehrere Arbeiten vor.

* Diss. Heidelberg 1952. Vorläufige Ergebnisse wurden auf der Physikertagung in Überlingen/Bodensee vorgetragen ${ }^{1}$.

${ }^{1}$ H. Faissner u. W. Guber, Physik. Verh. 3, 20 [1952].

${ }^{2}$ L. Puccianti, Nuovo Cimento 2, 257 [1907]; Mem. Spettroscop. 33, 133 [1904].

${ }^{3}$ D. Roschdestwensky, Ann. Physik 39, 307 [1912].

4 D. Roschdestwensky, Trans. opt. Inst. Lenin$\operatorname{grad} 13,2$ [1926].

5 A. Fillipov u. W. Prokofjew, Z. Physik 56, 458 [1929].

${ }^{6}$ A. Fillipov, Z. Physik 69, 526 [1931].

' R. Ladenburg, Z. Physik 48, 15 [1928].

${ }^{8}$ H. Kopfermann u. R. Ladenburg, Z. Physik 48, 26 [1928].
Mit der anomalen Dispersion elektrisch angeregter Gase befaßten sich Ladenburg, Kopfermann und Mitarbb. ${ }^{7-13}$ in umfangreichen Untersuchungen.

Dagegen ist die an den Einzellinien eines Bandenspektrums auftretende anomale Dispersion bisher kaum untersucht worden. Ältere Beobachtungen an den im Sichtbaren gelegenen Banden des Kupfers und der Erdalkalien ${ }^{14,15}$ hatten nur ergeben, daß eine Bande als Ganzes eine Anomalie des Brechungsindex verursacht. Es lag nahe, sie durch einen Summationseffekt der an den Einzellinien bewirkten Anomalien zu erklären. Zum Beweis suchten Julius und van der Plaats ${ }^{16}$ den anomalen

${ }^{9}$ H. Kopfermann u. R. Ladenburg, Z. Physik 48, 51 [1928].

10 A. Carst u. R. Ladenburg, Z. Physik 48, 192 [1928].

${ }^{11}$ H. Kopfermann u. R. Ladenburg, Z. Physik 65, 167 [1930].

12 R. Ladenburg u. S. Levy, Z. Physik 65, 189 [1930].

13 S. Levy, Z. Physik 72, 578 [1931].

14 F. Schön, Z. wiss. Photogr., Photophysik Photochem. 5, 349 [1907].

${ }^{15}$ H. Geisler, Z. wiss. Photogr., Photophysik Photochem. 7, 89 [1909].

${ }^{16}$ W. H. Julius u. B. J. van der Plaats, Z. wiss. Photogr., Photophysik Photochem. 10, 62 [1912]. 
Gang des Brechungsindex an den Einzellinien von Absorptionsbanden direkt nachzuweisen. Er zeigte sich angedeutet an den Linien des $\mathrm{NO}_{2}$; bei Bromund Joddampf dagegen war nur die Wirkung der ganzen Banden festzustellen. King ${ }^{17}$ wies schließlich in seinem mit einer Dispersion von ca. $1 \AA / \mathrm{mm}$ gewonnenen Aufnahmen die an den Einzellinien der Cyanbande (3838 $\AA$ ) auftretende anomale Dispersion deutlich nach. Die anomale Dispersion an ganzen Banden wurde ferner beiläufig in einigen der oben erwähnten Arbeiten von Roschdestwensky und Mitarbeitern festgestellt ${ }^{3,6}$. Darüber hinaus hat Roschdestwensky, wie er in anderem Zusammenhang mitteilte ${ }^{18}$, bereits bei den ersten Untersuchungen mit der Hakenmethode die in Ziff. 5 näher beschriebenen Bögen beobachtet.

Quantitative Messungen wurden schon früh an den ultraroten Absorptionsbanden von $\mathrm{CO}_{2}$ und $\mathrm{CO}$ vorgenommen ${ }^{19,20}$; auch sie beziehen sich aber auf die Wirkung der Banden als Ganzes. Dasselbe gilt von einer Arbeit Segrès ${ }^{21}$, in der die Dispersion von Joddampf über einen großen Teil des sichtbaren Spektrums verfolgt wurde. Auch bei einer Untersuchung von Ladenburg und Wolfssohn ${ }^{22}$ an den Schumann-Banden des $\mathrm{O}_{2}$ konnte nur die Wirkung der ganzen Bande nachgewiesen und eine ungefähre Abschätzung der den Einzellinien zugeordneten Oszillatorenstärken gegeben werden. Die einzige quantitative Untersuchung über die an den Einzellinien einer Bande auftretende anomale Dispersion scheint die von Ladenburg und Levy ${ }^{23}$ an den roten $\mathrm{Li}_{2}$-Banden durchgeführte zu sein.

Es war das Ziel der vorliegenden Untersuchung, möglichst quantitative Ergebnisse über die mit den Einzellinien von Bromdampf verbundene anomale Dispersion zu liefern. Aus experimentellen Gründen blieb sie auf den grüngelben Teil des Spektrums beschränkt. Insbesondere sollte die Proportionalität des Effekts mit der Dichte und seine Abhängigkeit von der Laufzahl der Bandenlinien nachgewiesen werden. In der Arbeit von Ladenburg und Le$\mathrm{vy}^{23}$ war ersteres aus prinzipiellen Gründen nicht möglich gewesen, während der Gang mit der Laufzahl innerhalb der Meßfehler lag. Weiter interessier-

${ }^{17}$ A. S. King, Astrophysic. J. 45, 254 [1917].

18 A. Afanasieff u. D. Roschdestwensky, Physik. Z. 14, 780 [1913].

${ }^{19}$ C. Statescu, Bull. Acad. Méd. Roumanie 3, 211 [1915]; Philos. Mag. 30, 737 [1915].

${ }^{20} \mathrm{~T}$. Wetterblad, Diss. Upsala 1924.

21 E. Segrè, Rend. Acad. Lincei (6) 10, 590 [1929]. ten die aus der anomalen Dispersion berechenbaren Oszillatorenstärken, da bisher über sie nur Abschätzungen aus Absorptionsmessungen bekannt waren.

Zugrunde gelegt wird die quantenmechanische Dispersionsformel $^{7}$ für $n-1 \ll 1$ und für Wellenlängen $\lambda$ (in $\mathrm{cm}$ ), die nahe den Wellenlängen der Linien $\lambda_{j k}$, jedoch außerhalb der eigentlichen Absorptionsgebiete liegen:

$$
n-1=\frac{r_{0}}{4 \pi} \sum_{j, k} \frac{\lambda_{j k}{ }^{3} \Re_{j k}}{\lambda-\lambda_{j k}} .
$$

Hierin bedeutet $r_{0}=e^{2} /\left(m c^{2}\right)$ den klassischen Elektronenradius (in $\mathrm{cm}$ ), $\mathfrak{\Re}_{j k}$ die ,Dichte (= Zahl pro $\mathrm{cm}^{3}$ ) der Dispersionselektronen für Übergänge von einem tieferen Zustand $k$ zu einem höheren $j$ “ im Sinne der klassischen Drudeschen Theorie.

In der quantentheoretischen Interpretation ist $\mathfrak{N}_{j k}$, solange man die ,negative Dispersion“ vernachlässigen kann, nur eine formale Abkürzung für das Produkt aus der Dichte der im $k$-ten Zustand befindlichen Molekeln $N_{k}$ und der Oszillatorenstärke $f_{j k}$

$$
\Re_{j k}=N_{k} f_{j k},
$$

wobei die Oszillatorenstärke $f_{j k}$ mit der Einsteinschen Übergangswahrscheinlichkeit $A_{j k}$ und dem Matrixelement $\left|\mathfrak{r}_{j k}\right|^{2}$ des Übergangs in bekannter Weise zusammenhängt ${ }^{12,24}$. Oft ist es bequem, die $\mathfrak{N}_{j k}$ proportional zur Gesamtdichte $N$ anzusetzen:

$$
\mathfrak{P}_{j k}=F_{j k} N \text {. }
$$

Wie man durch Vergleich mit (2) sieht, unterscheidet sich die Größe $F_{j k}$ von der Oszillatorenstärke $f_{j k}$ nur durch $N_{k} / N . \Re_{j k}$ ist aus dem Verlauf des Brechungsindex unmittelbar zu entnehmen. Da die Dichte des Bromdampfs leicht gemessen werden kann, ist damit auch $F_{j k}$ und wegen der bekannten Energiestufen der $\mathrm{Br}_{2}$-Molekel ${ }^{25,26}$ ebenso die Oszillatorenstärke $f_{j k}$ bekannt.

\section{Das sichtbare Absorptionsspektrum von Bromdampf}

Das Hauptabsorptionssystem von Bromdampf erstreckt sich von ungefähr $8000 \AA$ mit zunehmender Intensität bis zu dem bei etwa $5100 \AA$ einsetzen-

${ }^{22}$ R. Ladenburg u. G. Wolfssohn, Z. Physik 79, 44 [1932].

${ }^{23}$ R. Ladenburg u. S. Levy, Z. Physik 88, 449 [1934].

24 A. Sommerfeld, Atombau u. Spektrallinien II, 2. Aufl. Vieweg 1939, S. 365 ff.

25 W. G. Brown, Physic. Rev. 38, 1179 [1931].

26 W. G. Brown, Physic. Rev. 39, 777 [1932]. 
den starken Kontinuum. In seinem langwelligsten Teil wird es von einem zweiten, schwächeren Absorptionssystem überlagert, das in der angelsächsischen Literatur als extreme red system bezeichnet wird. Die Einzelbanden der Systeme sind nach Rot hin abschattiert und in Bandenzügen angeordnet. Die vollständige Schwingungsanalyse der sichtbaren Absorptionsbanden gelang erstmals Brown ${ }^{25}$, der auch die Rotationsanalyse des Hauptsystems durchführte ${ }^{26}$. Die Untersuchungen über das im fernen Roten gelegene System wurden später von Darby shire ${ }^{27}$ ergänzt. Im Rahmen der vorliegenden Arbeit können wir uns auf die Betrachtung des Hauptsystems beschränken.

Das Hauptabsorptionssystem kommt durch einen Elektronenübergang $0_{\mathrm{g}}^{+} \rightarrow 0_{\mathrm{u}}^{+}$zustande, was bei normaler Kopplung einen Übergang ${ }^{1} \Sigma_{\mathrm{g}}^{+} \rightarrow{ }^{3} \Pi_{0^{+}} \mathrm{u}$ entspräche ${ }^{27-29}$. Der Q-Zweig tritt daher nicht auf. Außerdem fällt bei den meisten grüngelben Banden jeweils die $J^{\prime \prime}$-te Linie des P-Zweigs mit der $\left(J^{\prime \prime}+3\right)$ ten des R-Zweigs zufällig fast zusammen ${ }^{26}$. $J$ ist dabei die Rotationsquantenzahl; der Doppelstrich bezieht sich wie auch im folgenden auf den unteren Elektronenzustand, während die auf den oberen Elektronenzustand bezüglichen Größen mit nur einem Strich gekennzeichnet seien. Die in Rede stehenden Banden machen den Eindruck, als ob sie nur aus einem Zweig bestünden. Erst bei starker Auflösung lassen sich diese sog. PR-Dubletts trennen.

Allerdings komplizieren sich die Verhältnisse durch die Isotopieverschiebung. Für die drei Molekelarten $\mathrm{Br}_{2}{ }^{79,79}, \mathrm{Br}_{2}{ }^{79,81}$ und $\mathrm{Br}_{2}{ }^{81,81}$ sind die Häufigkeiten nahezu 1:2:1. Die Linien der selteneren Molekelarten sind gegenüber den entsprechenden des $\mathrm{Br}_{2}{ }^{79,81}$ verschoben. Die Richtung der Verschiebung ist für die beiden Molekelsorten entgegengesetzt, ihr Betrag praktisch gleich ${ }^{25}$. Bei den gelbgrünen Banden ist er für Rotationsquantenzahlen um 50 nahezu gleich der Hälfte des Abstands von einem PR-Dublett zum nächsten. Ungefähr in der Mitte zwischen zwei vom $\mathrm{Br}_{2}{ }^{79}, 81$ herrührenden liegen also noch die beiden, den anderen Molekelisotopen angehörenden PR-Dubletts. Bei diesen ist jeweils die eine Komponente etwa halb so intensiv wie die entsprechende des $\mathrm{Br}_{2}{ }^{79,81}$, die andere wegen des bei Molekeln mit

${ }^{27}$ O. Darbyshire, Proc. Roy. Soc. [London], Ser. A 159, 93 [1937].

${ }^{28}$ P.Venkateswarlu, Proc. Indian Acad. Sci., Sect. A 24, 480 [1946].

${ }^{29}$ R. K. Asundi u. P. Venkateswarlu, Indian gleichen Kernen auftretenden Intensitätswechsels sehr schwach. Das tatsächlich beobachtete Bild ist noch komplizierter, da sich jeder Bande noch Linien hoher Laufzahl von benachbarten kurzwelligeren Banden und zusätzlich oft noch Banden aus anderen Bandenzügen überlagern.

Die Intensitätsverteilung innerhalb einer $\mathrm{Ab}$ sorptionsbande ist praktisch nur durch die thermische Verteilung der Molekeln auf die Rotationszustände des Ausgangs-Schwingungszustands bestimmt (s. etwa Herzberg ${ }^{30}$ S. 204 ff.). Diese ist bekanntlich ungefähr eine Maxwell-Verteilung. Das Maximum liegt für die ersten beiden Schwingungsquantenzahlen $v^{\prime \prime}=0$ und 1 bei der Rotationsquantenzahl $J^{\prime \prime}=36$ und verschiebt sich auch für höhere $v^{\prime \prime}$ nur wenig.

Die Intensitätsverteilung im Hauptsystem ergibt sich aus dem Franck-Condon-Prinzip. Darbyshire ${ }^{27}$ hat die Potentialkurven von Grund- und angeregtem Zustand aus den Brownschen Daten ${ }^{25,}{ }^{26}$ berechnet (s. ${ }^{27}$ Abb. 1). Da die Kurven stark gegeneinander verschoben sind, resultieren ausgeprägte Bandenzüge mit jeweils konstantem $v^{\prime \prime}$. Nach der halbklassischen Betrachtungsweise gingen die wahrscheinlichsten Übergänge, wie Darbyshire sie auch einzeichnet, von den Umkehrpunkten der klassischen Bewegung im Elektronengrundzustand zu den senkrecht darüberliegenden Punkten der oberen Potentialkurve. Die Bestimmung der wahrscheinlichsten Übergänge nach der Quantenmechanik nimmt man am einfachsten mit Hilfe der bei Herzberg ( $\mathrm{s}^{30} \mathrm{~S} .93$ ) angegebenen Eigenfunktionen des anharmonischen Oszillators auf graphischem Wege vor. Man erhält so für den Bandenzug $v^{\prime \prime}=1$ ein Intensitätsmaximum bei $v^{\prime} \approx 21$, während der Bandenzug $v^{\prime \prime}=0$ sein Intensitätsmaximum erst im Kontinuum annimmt. Natürlich nimmt die mittlere Intensität der Bandenzüge mit wachsendem $v^{\prime \prime}$ wegen der thermischen Verteilung auf die Schwingungszustände des Elektronengrundzustands stark ab.

\section{Messungen nach der Hakenmethode}

Die allgemeine Anordnung ist in Abb. 1 gegeben. Die Jaminröhre $R_{1}$ diente zur Aufnahme des Gases, $R_{2}$ blieb evakuiert. Die im Strahlengang des zweiten

J. Physics Proc. Indian Assoc. Cultivat. Sci. 21, 11 [1947].

${ }^{30}$ G. Herzberg, Molecular Spectr. and Molecular Structure I, Spectra of Diatomic Molecules, Sec. Edition, Toronto, etc. 1951. 
Bündels befindliche planparallele Glasplatte G konnte verstellt und ausgewechselt werden und diente dazu, die Ordnung des Interferenzstreifensystems beliebig zu erhöhen. Die Apparatur war erschütterungsfrei aufgebaut. Als Lichtquelle diente zuerst eine Quecksilber-Hochdrucklampe von Heraeus, später eine Xenon-Hochdrucklampe der Firma Osram. Letztere hatte eine etwas geringere Leuchtdichte, dagegen den Vorteil eines in dem untersuchten Spektralbereich praktisch völlig linienfreien Kontinuums.

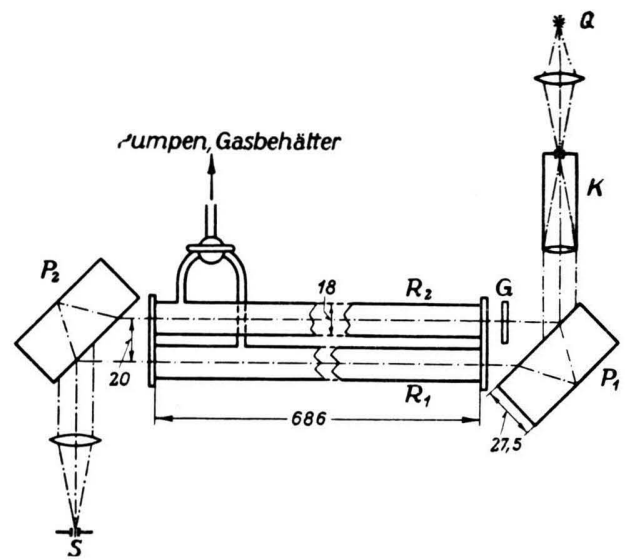

Abb. 1. Versuchsanordnung (Skizze nicht maßstabtreu; angegebene Maße in $\mathrm{mm}$ ); $\mathrm{Q}=$ Lichtquelle, $\mathrm{K}=$ Kollimator, $\mathrm{P}_{1}$ und $\mathrm{P}_{2}=$ Platten des Jaminschen Interferometers, $\mathbf{S}=$ Spektrograph.

Das verwendete Brom war ein chemisch reines Präparat der Firma Merck. Es befand sich in einem kleinen, mit der Jaminröhre $R_{1}$ über einen Glashahn verbundenen Kölbchen. Durch langzeitiges Abpumpen des über dem flüssigen Brom stehenden Dampfes mit der Wasserstrahlpumpe, sowie Ausfrieren der Probe mit flüssiger Luft und hohes Evakuieren des ganzen Röhrensystems wurde für möglichste Reinheit des Bromdampfes gesorgt. Der Druck wurde mit einem einfachen Quecksilber-Manometer gemessen, dessen Füllung mehrfach erneuert wurde. Die Temperatur wurde an der Außenwand der Jaminröhre mit einem gewöhnlichen Quecksilber-Thermometer festgestellt. Sie betrug bei allen Messungen etwa $20^{\circ} \mathrm{C}$.

Der Spektrograph war ein Apparat von Zeiß mit einem Reflexionsgitter von $3,3 \times 3,3 \mathrm{~cm}^{2}$ Fläche und 110 Strichen $/ \mathrm{mm}$. Der auflösbare Linienabstand errechnet sich daraus für eine Wellenlänge von $5400 \AA$ in der zu den Aufnahmen ausschließlich verwendeten 3. Ordnung zu $0,5 \AA$. Dies wurde bei Eichaufnahmen des Eisenspektrums ${ }^{31}$ auch erreicht. Bei den schrägliegenden Interferenzstreifen (Abb. 2) konnten $0,2 \AA$ auseinanderliegende Verzerrungen noch gut getrennt werden. Die Dispersion des Spektrographen ergab sich in dem photographierten Gebiet zwischen 5300 und $5900 \AA$ aus der Eichkurve graphisch zu im Mittel $9 \AA / \mathrm{mm}$.

${ }^{31}$ A. Gatterer u. J. Junkes, Arc Spectrum of Iron, Sec. Ed. Spec. Vaticana 1947.
Das spektral zerlegte Interferenzstreifensystem wurde in dem genannten Spektralbereich bei Dicken der Glasplatte $\mathrm{G}$ von $0,2,4,6$ und $8 \mathrm{~mm}$ und Bromdrucken zwischen 0 und 150 Torr photographiert. Aus Intensitätsgründen wurden die Aufnahmen bei einer Spaltbreite entsprechend $0,31 \AA$ gemacht. Das störende Violett der 4. Ordnung wurde dabei durch vorgesetzte Farbfilter von Schott beseitigt. Eine besonders feinkörnige Silber-Eosin-Platte von Perutz gab bei Entwicklung mit Rodinal die besten Ergebnisse. Die Belichtungszeit betrug in der Regel drei Min., gelegentlich auch zehn.

Ohne die Glasplatte G (Abb. 1) gibt der Verlauf der horizontal einjustierten Interferenzstreifen direkt den spektralen Gang des Brechungsindex des eingefüllten Gases wieder ${ }^{1,14-17}$. Durch Einbringen der Glasplatte erhalten die Interferenzstreifen eine zusätzliche Neigung gegen die $\lambda$-Achse (s. Abb. $2^{*}$ ), so daß sich nun auf der einen Seite einer isolierten Spektrallinie ein Minimum, auf der anderen ein Maximum im Streifenverlauf ausbildet. Aus dem spektralen Abstand $\triangle$ dieser Extrema, der sog. $\mathrm{Ha}$ ken, berechnet sich die durch die Gln. (1) und (2) definierte Dispersionselektronendichte $\mathfrak{N}_{j k}$ nach der Formel $^{3}$ :

$$
\mathfrak{N}_{j k}=\frac{\Delta^{2}}{\lambda_{j k}{ }^{3}} \frac{\pi}{r_{0}} \frac{K}{x},
$$

$x$ Schichtdicke des Gases, $K$ eine apparative Größe, in die Dicke und Brechungsindex der Glasplatte und damit auch die Wellenlänge eingehen. Sie läßt sich nach dem Vorgehen von Roschdestwensky ${ }^{3}$ durch Abzählen der Interferenzstreifen und Messen der zugehörigen Wellenlängendifferenz experimentell bestimmen.

Die Haken waren unter den vorliegenden experimentellen Verhältnissen wegen der kleinen Oszillatorenstärken und Linienabstände nicht sehr deutlich ausgeprägt. Am günstigsten erwies sich ein zusätzlicher Gangunterschied von $6 \mathrm{~mm}$ Glas, der für alle ausgewerteten Aufnahmen beibehalten wurde. Der Bromdruck durfte 30 Torr nicht übersteigen, da sonst die von benachbarten Linien herrührenden Verzerrungen ineinander flossen. Auch unterhalb dieses Druckes setzen sich die individuellen Verzerrungen für das Auge des Betrachters sofort zu den in Ziff. 5 näher zu betrachtenden Schattenfiguren zusammen.

Unter dem Mikroskop eines Zeiß'schen Komparators konnten jedoch die meisten der von den stärkeren Linien herrührenden Haken gut voneinander

* Abb. 2 und Abb. 5, s. Tafel S. 638 a. 
getrennt werden. Die Vermessung der Hakenabstände wurde aus den genannten Gründen nur an den Linien des häufigsten Molekelisotops $\mathrm{Br}_{2}{ }^{79,81}$ vorgenommen. Die Linien wurden auf $\pm 0,2 \AA$ genau identifiziert. Wenn der Mittelwert der beiden Hakenwellenlängen stärker von der aus den Molekülkonstanten berechneten Wellenlänge abwich, wurde die Messung nicht zur Auswertung herangezogen.

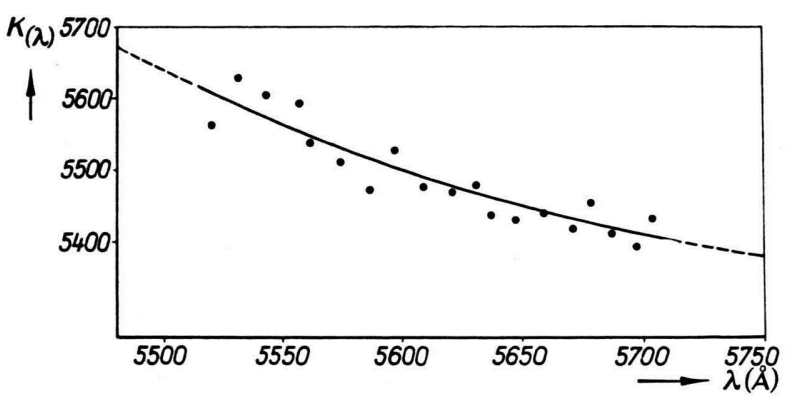

Abb. 3. Die apparative Größe $K$ als Funktion der Wellenlänge. sungen gültigen $K$ unterscheiden sich von dem gezeichneten nur wenig. Der Einfluß von Spaltbreite und Intensität der Lichtquelle auf die Ergebnisse konnte wegen der geringen Meßgenauigkeit nicht näher nachgeprüft werden. Wir können uns in diesem Punkt aber auf die eingehenden Untersuchungen von Ladenburg, Kopfermann u. a. ${ }^{7-13}$ stützen, die eine sehr weitgehende Unabhängigkeit der Ergebnisse von den angeführten Parametern ergeben hatten.

4. Ergebnisse der direkten Ausmessung der Hakenabstände

In Tab. 1 und 2 sind die gemessenen Hakenabstände aufgeführt. Damit der Gang mit derRotationsquantenzahl trotz der relativ großen Meßfehler deutlich hervortritt, wurden die gemessenen Werte in der angegebenen Weise zu Gruppen zusammengefaßt und darüber gemittelt. In jeder Gruppe sind mindestens sechs Messungen vereinigt. Der angegebene Fehler ist der mittlere Fehler der Mittelwerte.

\begin{tabular}{|c|c|c|c|c|c|c|c|c|}
\hline 1 & 2 & 3 & 4 & 5 & 6 & 7 & 8 & 9 \\
\hline \multirow{2}{*}{$\begin{array}{c}\text { Bande } \\
v^{\prime}-v^{\prime \prime}\end{array}$} & \multirow{2}{*}{$\begin{array}{c}\text { Torr } \\
\mathrm{Br}_{2}\end{array}$} & \multicolumn{7}{|c|}{$\Delta \cdot 10^{3}(\AA)$ für Rotationsquantenzahlen $J^{\prime \prime}$ von } \\
\hline & & $31-35$ & $36-40$ & $41-45$ & $46-50$ & $51-55$ & $56-60$ & $61-70$ \\
\hline \multirow[t]{2}{*}{$13-1$} & 16 & $178 \pm 9$ & $204 \pm 11$ & $194 \pm 8$ & $175 \pm 8$ & $186 \pm 11$ & $183 \pm 15$ & $168 \pm 9$ \\
\hline & 24 & - & - & \multicolumn{2}{|c|}{$228 \pm 6$} & \multicolumn{2}{|c|}{$230 \pm 17$} & - \\
\hline \multirow[t]{3}{*}{$14-1$} & 10 & $143 \pm 12$ & $176 \pm 18$ & $148 \pm 9$ & $159 \pm 3$ & \multicolumn{2}{|c|}{$149 \pm 9$} & - \\
\hline & 16 & $201 \pm 8$ & $213 \pm 7$ & \multicolumn{2}{|c|}{$199 \pm 8$} & \multicolumn{2}{|c|}{$166 \pm 10$} & 一 \\
\hline & 24 & - & - & - & - & \multicolumn{2}{|c|}{$227 \pm 14$} & 一 \\
\hline
\end{tabular}

Tab. 1. Gemessene Hakenabstände $\Delta$ in den Banden $13-1$ (5763 $\AA$ ) und 14-1 (5725 $\AA$ ) des $\mathrm{Br}_{2}{ }^{79,81}$ bei verschiedenen Bromdrucken.

\begin{tabular}{|c|c|c|c|c|c|c|c|c|}
\hline 1 & 2 & 3 & 4 & 5 & 6 & 7 & 8 & 9 \\
\hline \multirow{2}{*}{$\underset{v^{\prime}-v^{\prime \prime}}{\text { Bande }}$} & \multirow{2}{*}{$\begin{array}{l}\lambda_{\mathrm{K}} \\
(\AA ̊)\end{array}$} & \multicolumn{7}{|c|}{$\Delta \cdot 10^{3}(\AA)$ für Rotationsquantenzahlen $J^{\prime \prime}$ von } \\
\hline & & $26-30$ & $31-35$ & $36-40$ & $41-45$ & $46-50$ & $51-55$ & $56-60$ \\
\hline $17-1$ & 5620 & $242 \pm 12$ & $235 \pm 17$ & $228 \pm 10$ & $253 \pm 30$ & $293 \pm 18$ & $247 \pm 25$ & $229 \pm 13$ \\
\hline $18-1$ & 5589 & $239 \pm 13$ & \multirow{2}{*}{\multicolumn{2}{|c|}{$\begin{array}{l}267 \pm 6 \\
252 \pm 18\end{array}$}} & 209 & $=13$ & \multicolumn{2}{|c|}{$227 \pm 8$} \\
\hline $15-0$ & 5586 & - & & & $204 \pm 14$ & $170 \pm 14$ & $174 \pm 7$ & $175 \pm 9$ \\
\hline
\end{tabular}

Tab. 2. Gemessene Hakenabstände $\Delta$ bei einem Bromdruck von 20 Torr in verschiedenen $\mathrm{Banden}^{\mathrm{des}} \mathrm{Br}_{2}{ }^{79}{ }^{81}$.

Die Größe $K$ wurde für jede Meßreihe nach dem oben erwähnten Roschdestwenskyschen Verfahren bestimmt. Als Beispiel ist in Abb. $3 \mathrm{~K}$ als Funktion der Wellenlänge aufgetragen, wie für die Messungen Tab. 2 und 4 gültig war. Die für die anderen Mes-
Zur Berechnung der Dispersionselektronendichten genügt die einfache Gl. (4) wegen des Einflusses der benachbarten Linien nicht. Der Einfluß der anderen Komponente eines Dubletts wurde deshalb streng, die Wirkung der weiter entfernten Linien einer 
Bande wenigstens näherungsweise berechnet. Der Gang der Rechnung ist im Anhang (Ziff. 8) angedeutet. Bei den in Tab. 1 aufgeführten Banden sind die PR-Dublettabstände zwei- bis dreimal so groß wie die Hakenabstände. Die Korrektur auf den Einfluß der anderen Dublettkomponente wurde mit Hilfe der ersten beiden Glieder von Gl. (21) durchgeführt. Das zweite Glied trug in keinem Fall mehr als $4 \%$ des unkorrigierten Wertes bei. Die so korrigierten $\mathfrak{N}_{j k}$-Werte betrugen 50 bis $80 \%$ der nach (4) berechneten Werte. Gl. (21) war auch noch bei der Bande 15 - 0 anwendbar, wo die Dublettabstände noch merklich größer sind als die Hakenabstände.
$18-1$ und $15-0$ um $50 \%$ erhöht. Sie betragen dann zwischen 15 und $25 \%$ der direkt nach (4) errechneten $\mathfrak{N}_{j k}$-Werte.

Die so erhaltenen Werte für $\mathfrak{\Re}_{j k}$ sind in Tab. 3 und 4 aufgeführt. Die angegebenen Fehler sind die mittleren Fehler der Mittelwerte, wobei außer der statistischen Abweichung der Einzelmessungen auch die zu $10 \%$ ihres Wertes abgeschätzte Unsicherheit der Korrektur berücksichtigt wurde.

Zur Nachprüfung der Druckabhängigkeit und zur Berechnung der Oszillatorenstärken wurden die in den Tab. 3 und 4 aufgeführten $\Re_{j k}$-Werte gemäß der aus den Molekülkonstanten ${ }^{25,26}$ errechneten

\begin{tabular}{|c|c|c|c|c|c|c|c|c|}
\hline 1 & 2 & 3 & 4 & 5 & 6 & 7 & 8 & 9 \\
\hline \multirow{2}{*}{$\underset{v^{\prime}-v^{\prime \prime}}{\text { Bande }}$} & \multirow{2}{*}{$\begin{array}{c}\text { Torr } \\
\mathrm{Br}_{2}\end{array}$} & \multicolumn{7}{|c|}{$\mathfrak{R}_{j k} \cdot 10^{-8}\left(\mathrm{~cm}^{-3}\right)$ für Rotationsquantenzahlen $J^{\prime \prime}$ von } \\
\hline & & $31-35$ & $36-40$ & $41-45$ & $46-50$ & $51-55$ & $56-60$ & $61-70$ \\
\hline \multirow[t]{2}{*}{$13-1$} & 16 & $(88 \pm 20)$ & $121 \pm 15$ & $112 \pm 11$ & $93 \pm 15$ & $106 \pm 16$ & $104 \pm 20$ & $95 \pm 16$ \\
\hline & 24 & - & - & \multicolumn{2}{|c|}{$146 \pm 20$} & \multicolumn{2}{|c|}{$162 \pm 30$} & - \\
\hline \multirow[t]{3}{*}{$14-1$} & 10 & $(64 \pm 15)$ & $87 \pm 20$ & $71 \pm 13$ & $78 \pm 8$ & \multirow{3}{*}{\multicolumn{2}{|c|}{$\begin{array}{c}72 \pm 9 \\
87 \pm 11 \\
143 \pm 21\end{array}$}} & - \\
\hline & 16 & $(80 \pm 15)$ & $101 \pm 10$ & \multicolumn{2}{|c|}{$104 \pm 10$} & & & - \\
\hline & 24 & - & - & - & - & & & - \\
\hline
\end{tabular}

Tab. 3. $\mathfrak{R}_{j k}$-Werte der Linien in den Banden $13-1(5763 \AA)$ und $14-1$ (5725 $\AA$ ) des $\mathrm{Br}_{2}{ }^{79},{ }^{81}$ bei verschiedenen Bromdrucken.

\begin{tabular}{|c|c|c|c|c|c|c|c|c|}
\hline 1 & 2 & 3 & 4 & 5 & 6 & 7 & 8 & 9 \\
\hline \multirow{2}{*}{$\underset{v^{\prime}-v^{\prime \prime}}{\text { Bande }}$} & \multirow{2}{*}{$\begin{array}{l}\lambda_{\mathrm{K}} \\
(\AA)\end{array}$} & \multicolumn{7}{|c|}{$\mathfrak{R}_{j k} \cdot 10^{-8}\left(\mathrm{~cm}^{-3}\right)$ für Rotationsquantenzahlen $J^{\prime \prime}$ von } \\
\hline & & $26-30$ & $31-35$ & $36-40$ & $41-45$ & $46-50$ & $51-55$ & $56-60$ \\
\hline $17-1$ & 5620 & $131 \pm 15$ & $123 \pm 20$ & $110 \pm 13$ & $132 \pm 35$ & $(174 \pm 25) !$ & $124 \pm 26$ & $100 \pm 15$ \\
\hline $18-1$ & 5589 & $114 \pm 15$ & \multirow{2}{*}{\multicolumn{2}{|c|}{$\begin{array}{c}153 \pm 30 \\
(46 \pm 15)\end{array}$}} & \multicolumn{2}{|c|}{$80 \pm 20$} & \multicolumn{2}{|c|}{$87 \pm 15$} \\
\hline $15-0$ & 5586 & - & & & $(60 \pm 10)$ & $65 \pm 14$ & $68 \pm 10$ & $73 \pm 12$ \\
\hline
\end{tabular}

Tab. 4. $\mathfrak{N}_{j}$-Werte der Linien bei einem Bromdruck von 20 Torr in verschiedenen $\mathrm{Banden}^{\mathrm{des}} \mathrm{Br}_{2}{ }^{79,81}$.

In den Banden $17-1$ und $18-1$ dagegen betragen sie nur wenige Hundertstel $\AA$, so daß man die Wirkung zweier praktisch zusammenfallenden Linien mil3t. Der für eine Linie gültige Wert ist also ungefähr die Hälfte des gemessenen; die genaue Berechnung ist unmittelbar aus (19) möglich.

Die Korrektur auf den Einfluß der weiter entfernten Linien derselben Bande wurde nach (24) und (25) abgeschätzt. Um auch noch die Linien überlappender Banden annähernd zu erfassen, wurden die hiernach berechneten Korrektionen im allgemeinen um $30 \%$, bei den überlappenden Banden thermischen Verteilung auf die Rotationsquantenzahl $J^{\prime \prime}=55$ reduziert und gemittelt. Die bei kleinen $J^{\prime \prime}$ gemessenen Werte wurden im allgemeinen wegen der dort größeren Störungen durch Nachbarlinien bei der Mittelung etwas schwächer gewertet.

Die aus Tab. 3 erhaltenen reduzierten Dispersionselektronendichten $\mathfrak{N}_{j k}$ sind in Abb. 4 als Funktion des Bromdrucks aufgetragen. Sie liegen, wie es sein muß, für beide Banden auf einer durch den Nullpunkt gehenden Geraden. Die aus den gemittelten $\mathfrak{N}_{j k}$-Werten berechneten Molekülkonstanten sind in Tab. 5 gegeben. Neben $F_{j k}=\mathfrak{N}_{j k} / N$ sind 
H.Faissner, Die anomale Dispersion an den Linien der gelbgrünen Bromdampfbanden (S.633).

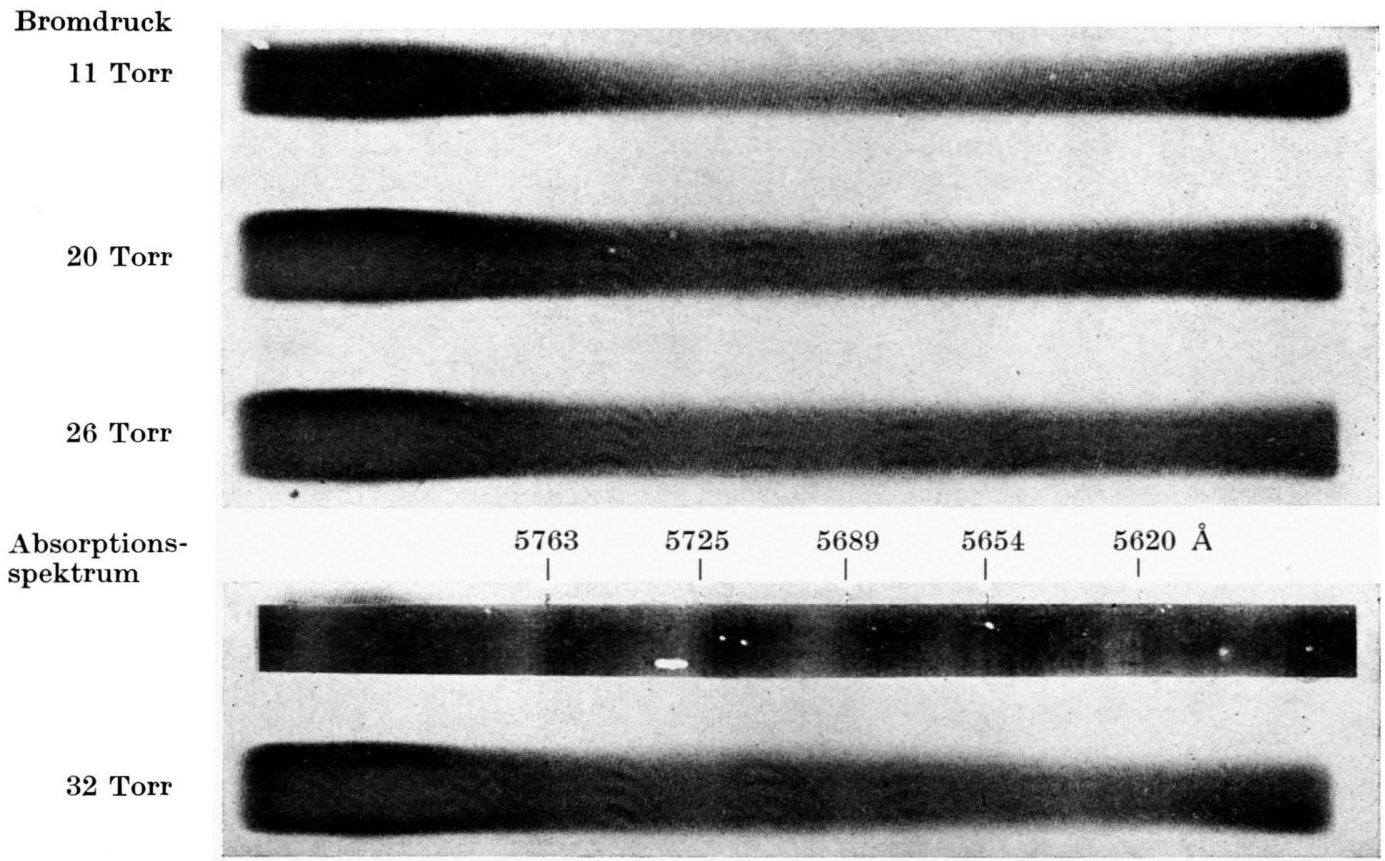

Abb. 2. Bögen zwischen 5800 und $5600 \AA$ (Photogr. Negativ, 4-fach vergr.). Die spektrale Intensitätsverteilung der Quecksilber-Hochdrucklampe wurde bei der Reproduktion auszugleichen versucht.

Bromdruck

$\approx 100$ Torr

71 Torr
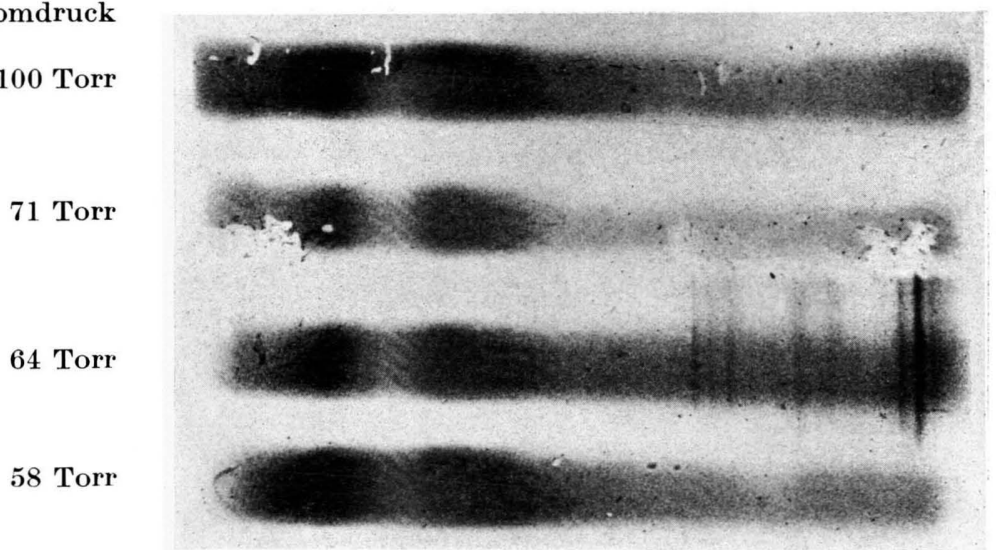

$$
\begin{array}{rlll}
\text { Bande: } & 13-1 & 14-1 & 15-1 \\
\lambda_{\mathrm{K}}: & 5763 & 5725 & 5689 \AA
\end{array}
$$

53 Torr

47 Torr

37 Torr

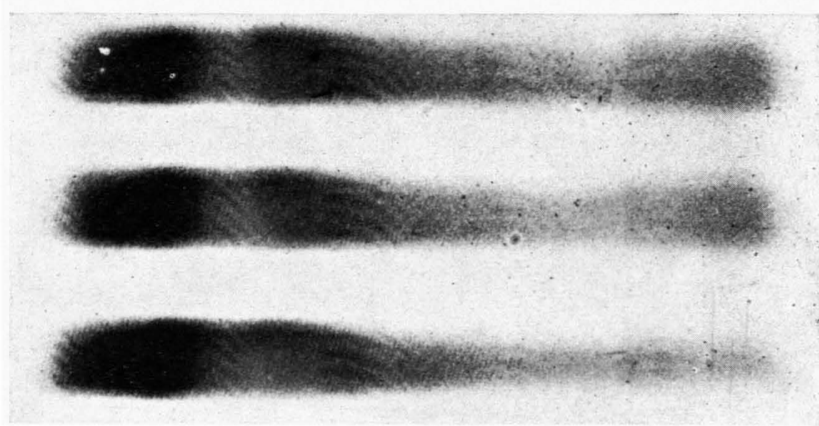

Abb. 5. Verschwinden der Bögen bei hohen Bromdrucken (Photogr. Negativ 4-fach). 

die Oszillatorenstärke $f_{j k}$, die Einsteinsche Übergangswahrscheinlichkeit $A_{j k}$ und das Matrixelement $\left|\mathfrak{r}_{j k}\right|^{2}$ aufgeführt. In den angegebenen mittleren Fehlern sind alle erfaßbaren Unsicherheiten berücksichtigt.

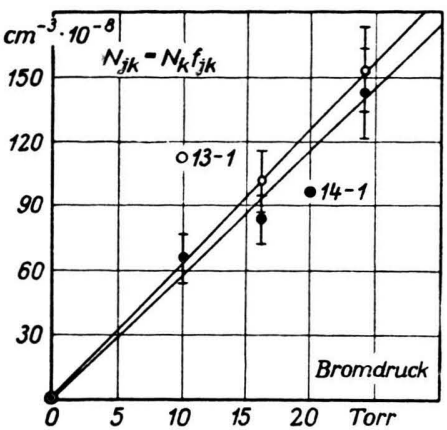

Abb. 4. Die reduzierten und gemittelten $\mathfrak{R}_{j k}$-Werte als Funktion des Bromdruckes.
Skizze (Abb. 6) angedeutet. Jedes Dublett ist dabei durch eine einzige Linie dargestellt.

Die Form der Bögen läßt sich unschwer berechnen. Man entnimmt der Abb. 6:

$$
\frac{\mathrm{d} y}{\mathrm{~d} \lambda}=\left(\frac{b}{a}-1\right) \operatorname{tg} \alpha .
$$

Rechnet man in der Deslandresschen Bandenformel die Laufzahl $\mu$ von der Bandenkante $\lambda_{\mathrm{K}}$ aus:

so wird

$$
1 / \lambda_{\mu}=1 / \lambda_{\mathrm{K}}-C \mu^{2},
$$

$$
a=\frac{\mathrm{d} \lambda_{\mu}}{\mathrm{d} \mu}=2 \lambda_{\mu}{ }^{2} V C\left(\frac{1}{\lambda_{\mathrm{K}}}-\frac{1}{\lambda_{\mu}}\right) .
$$

Die einfache Integration von (5) liefert damit $\left(\lambda_{\mu} \equiv \lambda\right)$ :

$$
y=\operatorname{tg} \alpha\left[b \sqrt{\frac{1}{C}\left(\frac{1}{\lambda_{\mathrm{K}}}-\frac{1}{\lambda}\right)}-\left(\lambda-\lambda_{\mathrm{K}}\right)\right] .
$$

\begin{tabular}{|c|c|c|c|c|c|}
\hline 1 & 2 & 3 & 4 & 5 & 6 \\
\cline { 2 - 5 } & $\begin{array}{c}\lambda_{\mathrm{K}} \\
(\AA)\end{array}$ & $\begin{array}{c}F_{j k}(55) \cdot 10^{8} \\
\begin{array}{c}\text { Bande } \\
v^{\prime}-v^{\prime \prime}\end{array}\end{array}$ & $\begin{array}{c}f_{j k} \cdot 10^{5} \\
-\end{array}$ & $\begin{array}{c}A_{j k} \cdot 10^{-3} \\
{\left[\mathrm{sec}^{-1}\right]}\end{array}$ & $\begin{array}{c}\left|\mathrm{r}_{j k}\right|^{2} \cdot 10^{21} \\
{\left[\mathrm{~cm}^{2}\right]}\end{array}$ \\
\hline $13-1$ & 5763 & $1,9 \pm 0,3$ & $1,7 \pm 0,3$ & $3,5 \pm 0,5$ & $9,2 \pm 1,4$ \\
$14-1$ & 5725 & $1,8 \pm 0,3$ & $1,6 \pm 0,3$ & $3,3 \pm 0,5$ & $8,4 \pm 1,3$ \\
$17-1$ & 5620 & $1,6 \pm 0,3$ & $1,5 \pm 0,2$ & $3,0 \pm 0,5$ & $7,5 \pm 1,2$ \\
$18-1$ & 5589 & $1,4 \pm 0,3$ & $1,3 \pm 0,3$ & $2,7 \pm 0,6$ & $6,5 \pm 1,4$ \\
$15-0$ & 5586 & $1,0 \pm 0,2$ & $0,19 \pm 0,05$ & $0,41 \pm 0,10$ & $0,98 \pm 0,23$ \\
\hline
\end{tabular}

Tab. 5. Durch direkte Ausmessung der Hakenabstände erhaltene Molekülkonstanten.

\section{Die Bogenmethode}

Wie in Ziff. 3 erwähnt, bilden sich in dem spektral zerlegten Interferenzstreifensystem hoher Ordnung bei Einfüllen des Bromdampfs charakteristische Bögen aus (Abb. 2). Sie werden mit wachsendem Bromdruck erst noch deutlicher und verschwinden schließlich wieder (Abb. 5). Auf dieses, durch die Druckverbreiterung der Absorptionslinien bedingte Verschwinden der Bögen läßt sich ein einfaches indirektes Verfahren zur Bestimmung der Oszillatorenstärken gründen.

Die Bögen kommen so zustande: Nach Ziff. 2 sind die Verzerrungen an den PR-Dubletts des $\mathrm{Br}_{2}{ }^{79,81}$ die weitaus größten. Da die Dubletts mit einem zur Bandenkante hin gleichmäßig abnehmenden Abstand angeordnet sind, setzen sich die bei jedem Schnitt eines PR-Dubletts mit einem der schräg liegenden Interferenzstreifen entstehenden Verdickungen für den Betrachter zu regelmäßigen Bögen zusammen. Das ist in der schematischen
Nahe der Kante geht dies über in

$$
y=\operatorname{tg} \alpha\left[\frac{b}{\lambda_{\mathrm{K}}} \sqrt{\frac{\lambda-\lambda_{\mathrm{K}}}{C}}-\left(\lambda-\lambda_{\mathrm{K}}\right)\right] .
$$

Der Bogen steigt also mit der Wurzel aus dem Wellenlängenabstand steil aus der Bandenkante heraus, erreicht, wie aus (5) ersichtlich, ein Maxi-

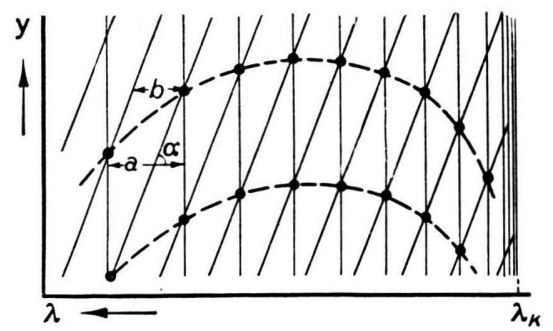

Abb. 6. Skizze zur Entstehung der Bögen.

mum bei $a=b$, fällt nach längeren Wellen hin langsamer $a b$ und geht asymptotisch in eine $\mathrm{zu}$ den Interferenzstreifen parallele Gerade über. 
Das Auftreten solcher Schattenfiguren ist schon früher beobachtet worden ${ }^{32}$. Wood stellte ähnliche Figuren bei der Untersuchung von Viellinienspektren im spektral zerlegten Interferenzstreifensystem eines Pérot-Fabry-Interferometers fest ${ }^{33}$ und deutete sie später im gleichen Sinn wie hier ${ }^{34}$. A fana sieff und Roschdestwensk ${ }^{18}$ fanden die Bögen mit einem Stufengitter im Absorptionsspektrum von Joddampf. Auch die Beobachtung der hier beschriebenen Bögen im spektral zerlegten Interferenzstreifenbild eines Jaminschen Interferometers wird in der genannten Arbeit erwähnt, doch wird kein Versuch unternommen, mit Hilfe der Bögen Aufschluß über die Stärke der anomalen Dispersion zu erhalten.

Das ist nun möglich, wenn man den Druck variiert und die Druckverbreiterung der Bromlinien heranzieht. Bei den hier vorliegenden Drucken und Temperaturen nimmt die Linienbreite gemäß der Lorentz-Weißkopfschen Stoßdämpfungstheorie linear mit dem Druck zu ${ }^{35-37}$, gleichgültig, ob man den Druck des betrachteten Gases selbst erhöht (Eigendruckverbreiterung), oder Fremdgas zusetzt (Fremdgasverbreiterung). Das ist durch die Absorptionsuntersuchungen von K ort üm und $\mathrm{Luck}^{38}$ erneut sichergestellt worden ${ }^{39-42}$. Da der Hakenabstand nach (4) nur der Wurzel aus dem Bromdruck proportional ist, rücken die Haken sowohl bei Vergrößerung des Eigendrucks als auch bei Zusatz von Fremdgas immer mehr in den Bereich starker Absorption und sind schließlich nicht mehr zu erkennen. Damit verschwinden natürlich auch die aus den Haken zusammengesetzten Bögen (Abb. 5). Der Bogen ist offenbar dann nicht mehr zu erkennen, wenn die am Ort der Haken herrschende Lichtintensität unter einen gewissen, sagen wir den $q$-ten Teil der Primärintensität abgesunken ist. $q$ wollen wir dabei in dem betrachteten relativ kleinen Wellenlängenbereich als konstant annehmen. $q$ läßt sich auf Grund der folgenden Rechnung aus den Daten jeder Meßreihe entnehmen.

Wir machen folgende vereinfachende Annahmen:

a) Die Intensität des einfallenden Lichts $I_{0}$ ist von der Wellenlänge unabhängig.

${ }^{32}$ Freundl. Hinweis von Herrn Dr. Luck ${ }^{38}$.

${ }^{33}$ R. W. Wood, Physik. Z. 8, 607 [1907].

${ }^{34}$ R. W. Wood, Physik. Z. 14, 681 [1913].

${ }^{35}$ V. Weißkopf, Z. Physik 75, 287 [1932].

${ }^{36}$ V. Wei $B$ kopf, Physik. Z. 34, 1 [1933].

${ }^{37}$ H. M. Foley, Physic. Rev. 69, 616 [1946].

${ }^{38}$ Herrn Dr. W. Luck danke ich verbindlich für die Übersendung eines Exemplares seiner Tübinger Disser- b) Die Linienbreite rührt nur von der Druckverbreiterung her.

c) Der Hakenabstand ist durch die einfache Gl. (4) gegeben.

d) Die Absorption rührt an der Stelle der Haken nur von der betrachteten Linie selbst her.

a) ist wegen der Kleinheit des betrachteten Wellenlängenintervalls gut erfüllt; b) ist wegen des starken Überwiegens der Druckverbreiterung über Dopplerverbreiterung und natürliche Linienbreite zulässig; c) und d) sind weitgehende Vernachlässigungen, die sich aber zum Teil kompensieren, da durch den Einfluß der Nachbarlinien sowohl der Hakenabstand als auch die Absorption an der Stelle der Haken vergrößert wird. Korrekturen, die man bei wenigstens teilweiser Berücksichtigung der Nachbarlinien anzubringen hat, werden anschlieBend berechnet.

Die nach Durchsetzen der Schichtdicke $x$ an der Stelle $\lambda$ beobachtete Intensität $I(\lambda)$ ist

$$
I(\lambda)=I_{0} e^{-\mu(\lambda) x} .
$$

Dabei ist der Absorptionskoeffizient ${ }^{36}$

$$
\mu\left(\lambda_{i}\right)=\frac{r_{0} \lambda_{1 / 2} \lambda_{j k}^{2} \Re_{j k}}{\left(\lambda-\lambda_{j k}\right)^{2}+\lambda_{1 / 2}{ }^{2}} .
$$

Die hierin auftretende Halbwertbreite $\lambda_{1 / 2}$, also der Abstand von der Linienmitte, bei dem der Absorptionskoeffizient auf die Hälfte seines Maximalwerts gesunken ist, wird nach der Stoßdämpfungstheorie ${ }^{36}$

$$
\lambda_{1 / 2}=\lambda^{2} S N(1) p .
$$

Hierin ist $S$ eine aus der Stoßdämpfungstheorie ${ }^{36}$ folgende Konstante der Dimension $\mathrm{cm}^{2}$, die unter anderem den optischen Wirkungsdurchmesser $\sigma$ enthält. $S_{\mathrm{b}}$ gelte für Stöße von Brommolekeln untereinander, $S_{\mathrm{f}}$ für Stöße von Fremdmolekeln gegen Brommolekeln. $N(1)$ ist die bei 1 Torr und der Beobachtungstemperatur in $1 \mathrm{~cm}^{3}$ vorhandene Molekelanzahl. $p$ ist der Gesamtdruck in Torr, $p_{\mathbf{b}}$ sei der Bromdruck.

Für den Abstand von der Linienmitte $\delta(q)$, bei dem die Intensität $I$ den $q$-ten Teil der Primärintensität beträgt, liefert (9) und (10)

tation schon vor der Publikation und für anregende und klärende Diskussionen.

${ }^{39}$ W. Lu ck, Diss. Tübingen 1951 u. Z. Naturforschg. 6 a, 191 [1951].

${ }_{40}$ G. Kortüm u. W. Luck, Z. Naturforschg. 6a, 306 [1951].

${ }^{41}$ W. Luck, Z. Naturforschg. 6a, 313 [1951].

${ }^{42}$ W. Luck, Z. Elektrochem. 56, 870 [1952]. 


$$
\delta(q)^{2}+\lambda_{1 / 2}^{2}=\frac{r_{0} \times \lambda_{1 / 2} \lambda_{j k}^{2} \Re_{j k}}{\log q} .
$$

Bei dem Grenzdruck $p$ bzw. $p_{\mathrm{b}}$, bei dem die Bögen gerade verschwinden, soll $\delta(q)$ gleich dem Abstand Haken-Linienmitte sein. Aus (4) und (12) folgt:

$$
\Re_{j k}\left\{\frac{r_{0} x}{\log q} \lambda^{2} \lambda_{1 / 2}-\frac{r_{0} x}{4 \pi K} \lambda^{3}\right\}=\lambda_{1 / 2}^{2} .
$$

Einsetzen von (11) ergibt für das gesuchte $F_{j k}=$ $\mathfrak{R}_{j k} / N$ die Formel für Eigendruckverbreiterung

$F_{j k}=\frac{S_{\mathrm{b}} \log q}{r_{0} x} \frac{1}{1-\frac{\log q}{4 \pi K \lambda S_{\mathrm{b}} N(1)} \frac{1}{p_{\mathrm{b}}}}$.

Bei Fremdgasverbreiterung rechnen wir so, als ob alle Stöße durch Fremdmolekeln verursacht würden, lassen also die etwas stärkere Wirkung der Brommolekeln außer acht. Das ist erlaubt, solange der Bromdruck nur einen Bruchteil des Gesamtdrucks beträgt und der Unterschied in der verbreiternden Wirkung nicht sehr groß ist. Wir erhalten so die Formel für Fremdgasverbreiterung

$$
F_{j k}=\frac{p}{p_{\mathrm{b}}} \frac{S_{\mathrm{f}} \log q}{r_{0} x} \frac{1}{1-\frac{\log q}{4 \pi K \lambda S_{\mathrm{f}} N(1)} \cdot \frac{1}{p}} .
$$

Wie man aus (14) sieht, nimmt bei Eigendruckverbreiterung der Einfluß des druckabhängigen Gliedes mit wachsendem Druck immer mehr ab. Die Konstante

$$
\frac{S_{\mathrm{b}} \log q}{r_{0} x}=F_{\mathrm{b}}
$$

hat offenbar die Bedeutung des kleinsten $F_{j k}$, bei dem noch ein Verschwinden des betreffenden Bogens erzielt werden kann. Die von noch schwächeren Banden herrührenden Bögen verschwinden über- haupt nicht mehr. Für Fremddruckverbreiterung ist die Grenze wegen $p / p_{\mathrm{b}}>1$ nach höheren $F_{j k}$ verschoben.

Da $F_{j k}$ eine positive Zahl ist, folgt aus (14) bzw. (15)

$$
p_{\mathrm{b}} \text { bzw. } p>\frac{\log q}{4 \pi K \lambda S N(1)}=p^{\mathrm{min}} .
$$

Man findet durch Umschreiben von (14):

$$
\left(p_{\mathrm{b}}-p_{\mathrm{b}}{ }^{\min }\right)\left(F_{j k}-F_{\mathrm{b}}\right)=p_{\mathrm{b}}{ }^{\min } F_{\mathrm{b}},
$$

also eine gleichseitige Hyperbel in der $\left(p_{\mathrm{b}}, F_{j k}\right)$ Ebene mit einem um $p_{\mathrm{b}}{ }^{\text {min }}$ und $F_{\mathrm{b}}$ verschobenen Achsenkreuz. Nimmt man die Untersuchung bei Fremdgaszusatz unter konstantem Verhältnis $p / p_{\mathrm{b}}$ vor, so ergibt sich dasselbe Bild, nur daß die Grenze nach höheren $F_{j k}$ verschoben ist. Während also unterhalb von $p^{\text {min }}$ die Drucksteigerung praktisch keinen Einfluß auf die Erkennbarkeit der Bögen hat, verschwinden bei Erreichen des Druckes $p^{\text {min }}$ fast gleichzeitig alle von den intensivsten Banden herrührenden Bögen. Dieses scharfe Einsetzen ermöglicht es, den Druck $p^{\text {min }}$ mit großer Genauigkeit experimentell zu bestimmen und daraus bei bekannten optischen Stoßdurchmessern die bisher noch offene Konstante $q$ zu errechnen.

Zur Korrektur der einfachen Formeln wird man vor allem die von der einen sehr benachbarten und praktisch gleich starken Linie herrührende Absorption mit in Rechnung ziehen und den Absorptionskoeffizienten additiv aus zwei Gliedern der Form (10) zusammensetzen. Führt man die Rechnung aus mit der für die meisten Banden noch zulässigen Näherung: $\lambda_{1 / 2}, \delta(q)$ klein gegen den PR-Dublettabstand $\lambda_{1}-\lambda_{2}$, so findet man die Gln. (14) und (15) als Spezialfälle enthaltende korrigierte Formel

$$
F_{j k}=\frac{F \cdot p / p_{\mathrm{b}}}{1-\frac{p^{\min }}{p}+\frac{r_{0} x \lambda^{3} N(1)}{4 \pi K} \frac{F_{j k}}{\left(\lambda_{1}-\lambda_{2}\right)^{2}} p_{\mathrm{b}}+\frac{S^{2} \lambda^{4} N(1)^{2}}{\left(\lambda_{1}-\lambda_{2}\right)^{2}} p^{2}}
$$

Hiernach sind auch die unmittelbar aus den gemessenen kleinsten Grenzdrucken entnommenen $p^{\text {min }}$ etwas zu korrigieren.

\section{Messungen und Ergebnisse nach der} Bogenmethode

Die Bögen wurden erst durch Erhöhung des Bromdrucks und später durch Zusatz von Luft oder vorzugsweise Wasserstoff zum Verschwinden gebracht.
Es erwies sich dabei als zweckmäßig, bei konstantem Mischungsverhältnis Bromdampf zu Wasserstoff den Gesamtdruck zu erniedrigen und das erste Auftauchen der Bögen zu beobachten. Es wurde im allgemeinen visuell und zwar in der zweiten Ordnung des Gitters beobachtet, gelegentlich zur Kontrolle auch photographiert. Die Spaltbreite hatte innerhalb weiter Grenzen auf die Erkennbarkeit der Bögen keinen Einfluß. Die Größe $K(\lambda)$ wurde aus Aufnahmen bestimmt, die bei evakuier- 
ten Jamin-Röhren vor, während und nach jeder Beobachtungsreihe gemacht wurden. Sie erwies sich jeweils als unverändert.

Tab. 6 gibt eine Auswahl der zum Verschwinden der Bögen führenden Grenzdrucke. Das nach längeren Wellen hin erst langsame, dann immer schnellere Anwachsen des Grenzdrucks bei Eigendruckverbreiterung erklärt sich aus der allgemeinen Zunahme der Intensität der Banden nach kurzen Wellen (Ziff. 2). Dies zeigt, daß der in Ziff. 5 unter vereinfachenden Annahmen abgeleitete Hyperbelverlauf die Verhältnisse in allen wesentlichen Zügen richtig wiedergibt.

\begin{tabular}{|c|c|c|c|c|}
\hline 1 & 2 & 3 & 4 & 5 \\
\hline \multirow[b]{2}{*}{$\begin{array}{c}\text { Bande } \\
v^{\prime}-v^{\prime \prime}\end{array}$} & \multirow[b]{2}{*}{$\begin{array}{l}\lambda_{\mathrm{K}} \\
(\AA)\end{array}$} & \multirow[b]{2}{*}{$\begin{array}{l}\text { Eigendruck } \\
\text { Reines Brom } \\
\text { (Torr) }\end{array}$} & \multicolumn{2}{|c|}{ Zusatz von $\mathrm{H}_{2}$} \\
\hline & & & $\begin{array}{c}\text { Gesamt- } \\
\text { druck } \\
\text { (Torr) }\end{array}$ & $\begin{array}{l}\text { Brom- } \\
\text { druck } \\
\text { (Torr) }\end{array}$ \\
\hline $36-0$ & 5169 & 15,0 & 70 & 7,5 \\
\hline $33-0$ & 5198 & 18,0 & 70 & 7,5 \\
\hline $30-0$ & 5237 & 20,0 & 48 & 7,0 \\
\hline $26-0$ & 5301 & 29,8 & 198 & 21,1 \\
\hline $24-0$ & 5340 & 33,4 & 160 & 23,2 \\
\hline $21-0$ & 5408 & 37,0 & 310 & 33,0 \\
\hline $19-0$ & 5461 & 41,6 & 406 & 43,2 \\
\hline $22-1$ & 5478 & 39,0 & 200 & 29,0 \\
\hline $19-1$ & 5559 & 63,0 & 540 & 57,5 \\
\hline $17-1$ & 5620 & $95 ?$ & 750 & 87,0 \\
\hline $13-1$ & 5763 & $115 ?$ & $>800$ & $>85$ \\
\hline $11-1$ & 6854 & $>150$ & $>800$ & $>85$ \\
\hline
\end{tabular}

Tab. 6. Zum Verschwinden der Bögen führende Drucke $p$ bei Eigen- und Fremddruckverbreiterung.

Die der ganzen Methode zugrunde liegende Hakenformel (4) hat zur Voraussetzung, daß die Linienbreite zu vernachlässigen ist. Um auch dies zu prïfen, wurden die Linien künstlich verbreitert, indem bei einem konstanten Bromdruck von 10--20 Torr Luft bis zu einem Gesamtdruck von 600 Torr zugesetzt wurde. Das Aussehen der Haken änderte sich hierdurch nicht merklich. Die Linienbreite ist also für die Dispersion in der Tat ohne Belang. Die beobachtete Absorption ist dagegen in einem viel größeren Abstand von der Linienmitte noch merklich, weil die große Schichtdicke eine starke zusätzliche Verbreiterung mit sich bringt.

$p^{\text {min }}$ betrug bei Eigendruckverbreiterung 13,6 Torr, bei Wasserstoffzusatz 50,0 Torr Gesamtdruck. Um hieraus $q$ nach Gl. (16) zu berechnen, braucht

${ }^{43}$ P. Schulz, Physik. Z. 39, 412 [1938].

${ }^{44} \mathrm{~W}$. Schütz, Schr. Königsberger GelehrtenGes. naturwiss. Kl. 16, 13 [1939]. man noch die optischen Stoßdurchmesser. Für optische Stöße gleichartiger Atome findet man in der Literatur allgemein Wirkungsdurchmesser von der rund 20-fachen Größe des gas-kinetischen Durchmessers angegeben ${ }^{36,43-45}$. Bei ungleichen Atomen beträgt der optische Stoßdurchmesser etwa das Doppelte des gaskinetischen ${ }^{36,43-46}$. Für die optischen Stoßdurchmesser von $\mathrm{Br}_{2}$ gegen Fremdgas differieren die experimentellen Daten bis zu einem Faktor 10 (vgl. $\left.{ }^{41}\right)$.

Es zeigte sich, daß unsere Messungen mit Wasserstoffzusatz am besten mit einem optischen Stoßdurchmesser $\sigma_{\mathrm{Br}_{2} \mathrm{H}_{2}} \approx 20 \AA \mathrm{zu}$ vereinbaren sind. Das ergibt einen Wert $q \approx 2$, also ein Verschwinden der Bögen, wenn die Lichtintensität an der Stelle der Haken auf die Hälfte der Primärintensität abgesunken ist. Behält man diesen Wert für die unter praktisch gleichen Bedingungen durchgeführten Beobachtungen mit Eigendruckverbreiterung bei, so erhält man nach (16) einen Stoßdurchmesser $\sigma_{\mathrm{Br}_{2} \mathrm{Br}_{2}}$ von $90 \AA$, der durchaus noch zu den oben erwähnten Ergebnissen bei artgleichen Atomen paßt. Die angegebenen Werte wurden für die Berechnung der $F_{j k}$ beibehalten. Es lassen sich jedoch unter Berücksichtigung der von den Nachbarlinien bewirkten Störungen und der Unsicherheit von $q$ auch noch Stoßdurchmesser $\sigma_{\mathrm{Br}_{2} \mathrm{Br}_{2}} \approx 60 \AA$ und $\sigma_{\mathrm{Br}_{2} \mathrm{H}_{2}} \approx 14 \AA$ mit unseren Messungen vereinbaren. Dagegen sind die von Kortüm und $\mathrm{Luck}^{40}$ abgeschätzten, gegenüber den gaskinetischen Durchmessern nur wenig vergrößerten Werte damit nur schwer verträglich, weil sie ein unplausibel kleines $q$ liefern würden.

In Tab. 7 ist eine Reihe der aus den gemessenen Drucken berechneten $F_{j k}$-Werte gegeben. Es sind jeweils die zwei vollständigsten Meßreihen mit Eigendruckverbreiterung und Zusatz von Wasserstoff aufgeführt. Das Verhältnis Gesamtdruck zu Bromdruck betrug bei der in Spalte 5 angegebenen Meßreihe 9,4 , bei der in Spalte 6 aufgeführten 6,8 . Andere, weniger systematische Beobachtungen führten zu praktisch damit übereinstimmenden Werten. Sind in Spalte 1 zwei Banden angegeben, so heißt das, daß die Ergebnisse für die erste, stärkere Bande, auf die sich die Tabellenwerte beziehen, durch eine überlagerte zweite Bande $u$. U. etwas gestört sein können.

${ }^{45}$ F. Waibel, Z. Physik 53, 459 [1929].

${ }^{46}$ Landolt-Börnstein, Zahlenwerte und Funktionen, 6. Aufl., Bd. I, 1, Springer-Verlag, Berlin 1950. 
Die Übereinstimmung der nach der gleichen Methode erhaltenen Werte ist recht gut, die zwischen Eigendruck- und Fremdgasmeßreihen befriedigend. Nur die bei den höchsten Drucken gewonnenen eingeklammerten Werte der Spalte 5 fallen systematisch aus dem Rahmen. Hier verlieren die mitgeteilten Formeln ihre Gültigkeit. Angaben über die Fehler sind angesichts der eingehenden Unsicherheiten schwer zu machen; doch sollten die Fehler zumindest der Relativwerte $15 \%$ nicht überschreiten.

\begin{tabular}{|c|c|c|c|c|c|}
\hline 1 & 2 & 3 & 4 & 5 & 6 \\
\hline \multirow{2}{*}{ Bande } & \multirow{2}{*}{$\begin{array}{l}\lambda_{\mathrm{K}} \\
(\AA)\end{array}$} & \multicolumn{4}{|c|}{$F_{j k} \cdot 10^{8}$ bestimmt mit } \\
\hline & & \multicolumn{2}{|c|}{ Eigendruck } & \multicolumn{2}{|c|}{$\mathrm{H}_{2}$-Zusatz } \\
\hline 34 bis $32-0$ & 5198 & 4,2 & 4,2 & - & 一 \\
\hline $31-0$ & 5223 & - & 3,5 & $(5,9)$ & - \\
\hline $30-0$ & 5237 & $(5,0)$ & 3,3 & 4,6 & - \\
\hline $29-0$ & 5250 & - & 3,0 & 3,3 & $(6,2)$ \\
\hline $28-0$ & 5266 & 2,5 & 2,7 & 3,1 & 3,7 \\
\hline $27-0$ & 5283 & 2,2 & 2,2 & 2,9 & 3,0 \\
\hline $26-0$ & 5301 & - & 2,0 & 2,8 & 2,7 \\
\hline 25 und $24-0$ & 5320 & 1,9 & 1,9 & 2,3 & 2,4 \\
\hline $23-0$ & 5362 & 1,9 & 1,9 & 2,0 & 2,4 \\
\hline $27-0$ & 5373 & - & 2,1 & 1,8 & 2,4 \\
\hline $22-0$ & 5385 & - & 1,9 & - & - \\
\hline $26-121-0$ & 5393 & 1,8 & 1,8 & 1,8 & - \\
\hline $25-11,0$ & 5412 & $.1,8$ & 1,8 & 1,7 & 2,0 \\
\hline $24-1\}^{20-0}$ & 5433 & 1,7 & 1,7 & 1,6 & - \\
\hline $23-1 \quad 19-0$ & 5456 & 1,7 & 1,7 & 1,4 & - \\
\hline $22-1$ & 5480 & 1,8 & 1,8 & - & - \\
\hline $21-1 \quad 18-0$ & 5505 & 1,7 & 1,7 & $(1,1)$ & 1,7 \\
\hline $20-1 \quad 17-0$ & 5532 & 1,7 & 1,6 & $(0,9)$ & 1,5 \\
\hline $19-1(16-0)$ & 5559 & 1,6 & 1,5 & $(0,9)$ & - \\
\hline $18-1$ & 5589 & 1,4 & 1,5 & $(0,5)$ & 一 \\
\hline $17-1$ & 5620 & - & 1,4 & $(0,5)$ & - \\
\hline $16-1$ & 5654 & - & 1,4 & - & - \\
\hline $15-1$ & 5689 & - & 1,3 & - & - \\
\hline $14-1$ & 5725 & - & 1,3 & - & - \\
\hline $13-1$ & 5763 & 一 & 1,3 & - & - \\
\hline
\end{tabular}

Tab. 7. Die Größe $F_{j k}=f_{j k} N_{k} / N$ nach der Bogenmethode.

\section{Diskussion}

Die deutliche Abhängigkeit der gemessenen Hakenabstände (Tab. 1 und 2) von der Rotationsquantenzahl $J^{\prime \prime}$ ist zum großen Teil durch den bei kleineren $J^{\prime \prime}$ stärkeren Einfluß der Nachbarlinien bedingt. Die $J^{\prime \prime}$-Abhängigkeit der $\mathfrak{l}_{j k}$-Werte (Tab. 3 und 4) liegt noch innerhalb der Meßfehler. Sie ist aber, wenn man die Gesamtheit der Messungen betrachtet, noch ausgeprägt und bei den am wenigsten durch überlappende Banden gestörten Messungen (Tab. 3) übereinstimmend von der nach der thermischen Verteilung zu erwartenden Größe. So kann wenigstens von einer qualitativen Bestätigung der theoretisch geforderten $J$-Abhängigkeit gesprochen werden. Die Druckabhängigkeit der gemittelten $\mathfrak{N}_{j k}$-Werte ist dagegen einwandfrei außerhalb der Meßfehler und zeigt die theoretisch geforderte Proportionalität (Abb.4). Die Änderung der Oszillatorenstärke von Bande zu Bande (Tab. 5) war bei der direkten Ausmessung innerhalb des $v^{\prime \prime}=1$ Bandenzugs nicht nachzuweisen. Ihre systematische Abnahme nach kurzen Wellen hin liegt noch knapp innerhalb der Meßfehler und ist wahrscheinlich durch die bei den kurzwelligen Banden stärkeren Störungen vorgetäuscht. Jedenfalls läßt sich aus den Messungen entnehmen, daß sich die Oszillatorenstärken der $v^{\prime \prime}=1$-Banden im untersuchten Spektralgebiet nur wenig ändern. Im Gegensatz dazu steigt die Intensität der $v^{\prime \prime}=0$-Banden mit zunehmender Schwingungsquantenzahl $v^{\prime}$ stark an. Die $\mathrm{Br}_{2}{ }^{79,81}$-Linien des $v^{\prime \prime}=0$-Bandenzugs erwiesen sich für $v^{\prime} \leqq 14$ als noch schwächer als die von den anderen Molekelisotopen herrührenden Linien der überlappenden $v^{\prime \prime}=1$-Banden. Dort wurden sie deshalb nicht systematisch ausgemessen und nicht tabelliert. Für $v^{\prime}=15$ sind sie dagegen bereits gut zu messen (Tab.4), mit $v^{\prime}=16$ annähernd gleich intensiv wie die dort gelegenen $\mathrm{Br}_{2}{ }^{79}{ }^{81}$-Linien des $v^{\prime \prime}=1$-Bandenzugs. Genau das ist nach dem Franck-Condon-Prinzip zu erwarten (Ziff. 2), das für den $v^{\prime \prime}=0$-Bandenzug einen Intensitätsanstieg bis zum Kontinuum, für den $v^{\prime \prime}=1$-Bandenzug ein Intensitätsmaximum bei $v^{\prime} \approx 21$ ergibt.

Die direkten Messungen werden bestätigt und ergänzt durch die nach der Bogenmethode erhaltenen Ergebnisse (Ziff. 6). Die Zunahme der $F_{j k}$-Werte nach kürzeren Wellen hin (Tab. 7) ist hier stark ausgeprägt. Extrapoliert man die gemessenen $F_{j k^{-}}$ Werte des $v^{\prime \prime}=0$-Bandenzugs linear bis zum Einsetzen des Kontinuums, so kommt man auf ein $F_{j k}$ von ungefähr $8,5 \cdot 10^{-8}$. Berücksichtigt man, daß der Anstieg in Wirklichkeit etwas stärker als linear geht und die Intensität im Kontinuum noch etwas weiter steigt ${ }^{47}$, so erhält man für das Verhältnis der maximalen $F_{j k}$ der Bandenzüge $v^{\prime \prime}=0$ und $v^{\prime \prime}=1$ ungefähr 5, und das ist mit guter Genauigkeit das Verhältnis der Besetzungszahlen im 0-ten und 1-ten Schwingungsquant des Elektronengrundzustandes. Das ist ein Hinweis darauf, daß die Wahrscheinlichkeiten für Übergänge vom 0-ten und 1-ten Schwin-

${ }^{47}$ C. Jaffé, Handbuch Exp. Physik, Bd. XIX, Leipzig 1928 . 
gungszustand aus nicht sehr verschieden sind. Bei den Messungen mit Eigendruckverbreiterung wurde bei der einzigen in diesem Gebiet nicht völlig von $v^{\prime \prime}=0$-Banden überlappten $v^{\prime \prime}=1$-Bande $22-1$ ein relatives Intensitätsmaximum beobachtet (Tab. 7), das gut zu der Abschätzung nach dem FranckCondon-Prinzip stimmen würde. Allerdings wird die Brauchbarkeit der Bogenmethode durch die bei kürzeren Wellen ziemlich starke Überlappung der verschiedenen Banden etwas beeinträchtigt. Wie die gute Übereinstimmung mit den direkt gemessenen Werten zeigt (Tab. 5 und 7), fiel dies jedoch bei den langwelligeren Banden nicht ins Gewicht.

Auch sind die gemessenen Absolutwerte der Oszillatorenstärke etwa von der nach der Theorie zu erwartenden Größe. Nach dem $f$-Summensatz von Thomas, Kuhn und Reiche ${ }^{24}$ sollte nämlich die Summe der Oszillatorenstärken $f_{j k}$ bei festem Ausgangszustand $k$ über alle möglichen Endzustände $j$ ungefähr gleich 1 sein. Da nur ein kleiner Bruchteil der möglichen Übergänge ausgemessen wurde, kann allerdings nur eine grobe Abschätzung gemacht werden. Diese führt unter Annahme eines mittleren $f_{j k}$ von $10^{-5}$ für das Hauptsystem und eines höchstens ebenso großen für die anderen Übergänge zu einer $f$-Summe für den diskreten Teil des Spektrums von höchstens ca. $10^{-2}$. Ein so kleiner Wert ist aber nach unșerer Kenntnis der Brommolekel $\mathrm{zu}$ erwarten, da nach den vorliegenden experimentellen $^{47}$ und theoretischen ${ }^{48}$ Ergebnissen der bei weitem überwiegende Teil der Absorption von Bromdampf vom Kontinuum herrührt.

Zum Vergleich mit den Ergebnissen anderer Autoren liegen nur wenige Arbeiten vor. An einzelnen Bandenlinien von $\mathrm{Li}_{2}$ haben Ladenburg und Le v y ${ }^{23} \mathfrak{R}_{j k}$-Werte gleicher Größenordnung mit ungefähr gleichen Fehlern ausgemessen. Oszillatorenstärken von gleicher Größe, wie sie in der vorliegenden Arbeit gefunden wurden, schätzten Ladenburg und Wolfssohn ${ }^{22}$ in den Schumann-Banden des $\mathrm{O}_{2}$ aus dem Verlauf der normalen Dispersion ab. Über die Linienstärken im diskreten Bromdampfspektrum scheinen nur Kortüm und $\mathrm{Luck}^{40} \mathrm{An}$ gaben gemacht zu haben. Sie schätzten aus ihren in Ziff. 5 besprochenen Absorptionsmessungen für die innerhalb der grünen Quecksilberlinie 5461 $\AA$ liegenden Bromlinien ein mittleres $F_{j k}$ von $5,2 \cdot 10^{-8}$ ab. Der Wert ist fast dreimal so groß wie der aus

${ }^{48}$ J. Mulliken, J. chem. Physics 7, 20 [1939]; 8, 234 u. 382 [1940]. den hier gegebenen Messungen folgende. Die Diskrepanz ist noch nicht ganz geklärt. Die Größenordnung wird jedenfalls von den beiden verschiedenen Methoden übereinstimmend geliefert.

8. Anhang zu Ziff. 4: Einfluß der Nachbarlinien auf die gemessenen Hakenabstände

Für ein Dublett mit gleich starken Komponenten erhält man analog der Roschdestwenskyschen Betrachtung ${ }^{3}$ als Bestimmungsgleichung der Hakenwellenlängen

$$
\frac{\Delta_{1}{ }^{2}}{4}\left\{\frac{1}{\left(\lambda-\lambda_{1}\right)^{2}}+\frac{1}{\left(\lambda-\lambda_{2}\right)^{2}}\right\}=1 .
$$

Dabei ist $\Delta_{1}$ der aus (4) zu entnehmende Hakenabstand, den man an einer isolierten Linie von der Stärke einer Dublettkomponente messen würde. Wenn der Dublettabstand $\lambda_{1}-\lambda_{2}$ kleiner ist als $\sqrt{2} \Delta_{1}$, so hat diese Gleichung 4 . Grades nur zwei reelle Wurzeln. Zwischen den Linien sind die Interferenzstreifen zwar noch verzerrt, es werden aber keine eigentlichen Haken mehr ausgebildet. Die gesuchte Größe $\Delta_{1}$ läßt sich bei bekanntem $\lambda_{1}$ und $\lambda_{2}$ aus jeder der beiden meßbaren Hakenwellenlängen berechnen.

Im Falle $\lambda_{1}-\lambda_{2}>\sqrt{2} \Delta_{1}$ existieren vier reelle Wurzeln, die mit $\lambda_{1}{ }^{\prime}, \lambda_{1}{ }^{\prime \prime} ; \lambda_{2}{ }^{\prime}, \lambda_{2}{ }^{\prime \prime}$ bezeichnet seien. Die bei einer isolierten Linie von der Linienmitte gleich weit entfernten Haken werden durch die Wirkung der zweiten Linie beide von der Linie weg verschoben. Die Verschiebung ist aber auf der von der zweiten Linie abgewandten Seite wegen des größeren Abstandes kleiner als auf der zugewandten, so daß nun die Haken in der in Abb. 7 angedeuteten Weise asymmetrisch zu jeder Linie gelegen sind.

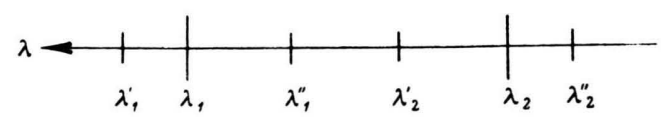

Abb. 7. Zur Lage der Haken bei einem PR-Dublett.

Um mit einer genauen Relativmessung auskommen zu können, leitet man zweckmäßig aus (19) einen Ausdruck für $\Delta_{1}$ her, der nur noch die Differenzen der gemessenen Haken- und berechneten LinienWellenlängen unter sich enthält.

Die Ableitung geht in großen Zügen so: Aus dem bekannten Zusammenhang der Konstanten einer algebraischen Gleichung mit ihren Wurzeln erhält man mit Hilfe der evidenten Symmetriebeziehung

$$
\lambda_{1}^{\prime}-\lambda_{1}^{\prime \prime}=\lambda_{2}{ }^{\prime}-\lambda_{2}{ }^{\prime \prime}=\Delta
$$


nach einigen Umformungen die quadratische Gleichung für $\Delta_{1}^{2}$

$$
\Delta_{1}^{4}+16\left(\lambda_{1}-\lambda_{2}\right)^{2} \Delta_{1}^{2}=4 \Delta^{2}\left(\lambda_{1}^{\prime}-\lambda_{2}{ }^{\prime}\right)^{2}
$$

und durch Entwickeln der in ihrer Lösung auftretenden Wurzel die Hakenformel für ein Dublett mit gleich starken Komponenten

$$
\Delta_{1}^{2}=\Delta^{2}\left\{\frac{\lambda_{1}{ }^{\prime}-\lambda_{2}{ }^{\prime}}{\lambda_{1}-\lambda_{2}}\right\}^{2}-\frac{1}{4} \Delta^{2}\left\{\frac{\Delta}{\lambda_{1}-\lambda_{2}}\right\}^{2}\left\{\frac{\lambda_{1}^{\prime}-\lambda_{2}{ }^{\prime}}{\lambda_{1}-\lambda_{2}}\right\}^{4}+-\ldots
$$

Um $\left(\lambda_{1}{ }^{\prime}-\lambda_{2}{ }^{\prime}\right) /\left(\lambda_{1}-\lambda_{2}\right)$ abzuschätzen ${ }^{49}$, setzt man die um $\lambda_{1}$ liegenden beiden Lösungen von (19) in der Form $\lambda=\lambda_{1}+\varepsilon$ an und vernachlässigt Glieder mit $\varepsilon^{3}$ und $\varepsilon^{4}$. Man findet so aus (19) in guter Näherung für das Verhältnis der beiden $\mathrm{Ab}$ stände

$$
\left|\varepsilon_{1} / \varepsilon_{2}\right|=1-\frac{\Delta_{1}}{\lambda_{1}-\lambda_{2}} .
$$

Daraus läßt sich bei bekanntem $\Delta_{1}$ der gesuchte Quotient berechnen. Da $\Delta_{1}$ von vornherein nicht zugänglich ist, setzt man in (22) den gemessenen Hakenabstand $\Delta$ als nullte Näherung ein und iteriert. Dieses Verfahren scheint umständlicher zu sein als die direkte Berechnung nach (21). Es ist jedoch vorzuziehen, wenn man bei nicht zu kleinem Korrektionsfaktor eine große Zahl von Messungen geringer Genauigkeit vorliegen hat. Dann wird man $\mathfrak{N}_{j k}$ einfach nach der Formel für eine isolierte Linie berechnen und nach der Mittelung eine auf dem beschriebenen Weg erhaltene Korrektur anbringen.

Der Einfluß der weiter entfernt liegenden Linien desselben Bandenzweiges ist viel kleiner. Charakterisieren wir die Linien der Kürze halber durch ihre Laufzahl $m$ in der Deslandresschen Bandenformel und bezeichnen wir die betrachtete Linie mit $m_{0}$, so lautet die vollständige Hakenformel

$$
\frac{\Re_{m_{0}} \lambda_{m_{0}}^{3}}{\left(\lambda_{m_{0}}-\lambda_{m_{0}}^{\prime}\right)^{2}}+\sum_{m \neq m_{0}} \frac{\Re_{m} \lambda_{m}^{3}}{\left(\lambda_{m}-\lambda_{m_{0}}\right)^{2}}=\frac{4 \pi}{r_{0}} \frac{K}{x} \text {. }
$$

Dabei ist in der Summe, in sicher erlaubter Näherung, $\lambda_{m_{0}}^{\prime}$ gleich $\lambda_{m_{0}}$ gesetzt.

49 Nach einem Vorschlag von Herrn Dr. O. Herrmann, dem ich auch sonst für Beratung bei den folgenden Abschätzungen und Unterstützung in der numerischen Rechnung zu Dank verpflichtet bin.
Da man ungefähr beim Maximum der Linienintensität mißt, gewinnt man eine obere Schranke für den Einfluß dieser Nachbarlinien, wenn man alle $\mathfrak{R}_{m} \lambda_{m}^{3}$ gleich $\mathfrak{N}_{m_{0}} \lambda_{m_{0}}^{3}$ setzt und die Summe über $m$ mit dem herausgezogenen Term vergleicht. Wegen der nach größerem $m$ zunehmenden Linienabstände wird die Summe über $m>m_{0}$ durch

$\frac{1}{\left(\lambda_{m_{0}}+1-\lambda_{m_{0}}\right)^{2}} \sum_{\nu=1}^{\infty} \frac{1}{v^{2}}=\frac{1}{\left(\lambda_{m_{0}}+1-\lambda_{m_{0}}\right)^{2}} \cdot \frac{\pi^{2}}{6}$

majorisiert, während man in der Summe über $m<m_{0}$ die Linienabstände nach der Deslandresschen Bandenformel einsetzen und die Summe durch ein Integral nach oben abschätzen kann. Man findet

$$
\begin{aligned}
\sum_{m<m_{0}} \frac{1}{\left(\lambda_{m}-\lambda_{m_{0}}\right)^{2}} \approx & \frac{1}{\left(\lambda_{m_{0}}-1-\lambda_{m_{0}}\right)^{2}} \\
& +\frac{1}{\lambda_{m_{0}}^{4} C^{2}\left(2 m_{0}\right)^{2}}
\end{aligned}
$$

wobei $\mathrm{C}$ die beim quadratischen Glied der Bandenformel stehende Konstante ist.

Aus diesen Korrektionen auf den Einfluß eines Bandenzweigs ist die Korrektion für die Wirkung aller Linien derselben Bande sofort zu berechnen. Die Wirkung der außerhalb der untersuchten Bande gelegenen Linien kann wegen ihres quadratischen Abfalls mit dem Wellenlängenabstand vernachlässigt werden; der Einfluß der von anderen Banden herrührenden überlappenden Linien ist rechnerisch schwer zu erfassen.

Meinem hochverehrten Lehrer, Herrn Prof. Dr. W. Bothe danke ich herzlich für die Anregung zu der vorstehenden Arbeit und ihre stete Förderung. Herrn Prof. Dr. H. Kopfermann bin ich für kritische Durchsicht.des Manuskripts, Herrn Dr. W. Guber für Beratung bei den optischen Fragen zu Dank verbunden. 